\title{
A Tool for Developing Collaborative Sensing and Cognitive MAC Layer Solutions for 5G in Rural Areas
}

\author{
G. Ferreira* ${ }^{*}$ P. Solis Barreto $^{\dagger}$, M. F. Caetano ${ }^{\dagger}$, E. Alchieri ${ }^{\dagger}$, J. Vartiainen ${ }^{\S}$, \\ H. Karvonen ${ }^{\S}$, M. Matinmikko-Blue ${ }^{\S}$, J. Seki ${ }^{\ddagger}$ \\ Departament of Computer Science, University of Brasilia, Brasilia, Brazil \\ Email: *gabrielcarvfer@aluno.unb.br, ${ }^{\dagger}\{$ pris,mfcaetano,alchieri\}@unb.br \\ Centre for Wireless Communications, Oulu University, Oulu, Finland \\ Email: ${ }^{\S}$ heikki.karvonen,johanna.vartiainen,marja.matinmikko\}@oulu.fi \\ Centro de Pesquisa e Desenvolvimento em Telecomunicacoes, CPQD, Campinas, Brazil \\ Email: $\ddagger_{j}$ jeki@ cpdq.br
}

\begin{abstract}
The 5G mobile networks will increase the amount of available spectrum by using new licensed and unlicensed bands. However, most of the newly allocated bandwidth is restricted to higher frequencies, that provides smaller coverage. Lower frequencies, that provide higher ranges, are mostly occupied by either radio or television channels. With the move to digital television, some analog TV channels are available in specific regions, opening the possibility of frequency reuse by third parties. Geolocation databases and collaborative sensing techniques are used to locate and use the available TV Whitespaces (TVWS) for mobile transmission. This paper presents the proposal of a cognitive cycle in the ns-3 simulator (LENA/LTE), which includes collaborative sensing and a cognitive radio resource scheduler. The simulations were done considering a rural area and the results show that the implementation may be useful for further research in developing new solutions for the 5G Cognitive MAC Layer.
\end{abstract}

Index Terms-5G, collaborative spectrum sensing, cognitive, MAC Layer, dynamic spectrum access, simulation, ns-3, rural areas.

\section{INTRODUCTION}

The rapid expansion of mobile connected devices along with new internet services generated a great demand for more spectrum. Increasing the available bandwidth and spectral efficiency is necessary to guarantee appropriate levels of service for different applications. It is expected that the next generation of mobile networks, 5G, will increase the network capacity in 1000 times, increasing both the data rates and number of concurrently connected devices from 10 and up to 100 times when compared to the current $4 \mathrm{G}$ networks.

Nowadays, most of the radio spectrum is allocated to different licensees that operate telecommunication services (satellite and mobile networks) along with other civil and military applications (e.g. air traffic control, radar and radio telescopes, TV and radio broadcast). The remaining fraction of unlicensed spectrum is shared by multiple technologies that coexist and typically usually use Carrier Sense Multiple Access (CSMA), a decentralized medium access control (MAC) protocol. The telecommunication licensed bands typically use a centralized
MAC protocol, preventing collisions, wait times and the exchange of control messages to establish the medium control (CSMA). The most common MAC protocols used for licensed channels are: Frequency-Division Multiple Access (FDMA), that divides the channel into frequency slices; Time-Division Multiple Access (TDMA), that divides the channel into time slices; and Orthogonal Frequency-Division Multiple Access (OFDMA), that divides the channel into resource blocks, that are time and frequency slices, known as Resource Blocks, providing more granularity and improving even further the spectrum utilization when compared to FDMA and TDMA.

Although more efficient MAC protocols increase the spectrum utilization of the bands, they are incapable to increase the amount of available spectrum to be allocated, due the fixed spectrum allocation policies [1].

Multiple studies show that the licensed spectrum bands are not fully used most of the time and mainly, further away from large cities [1]-[3]. The Cognitive Radio (CR) technology is a proposal to increase spectral efficiency and spectrum utilization. One of the $\mathrm{CR}$ techniques used to improve the licensed spectrum bands utilization is the Dynamic Spectrum Access (DSA), where non-licensees, known as Secondary Users (SU), use the bands of the licensee, known as Primary User (PU), without disturbing their transmissions. The SUs should start transmitting only while the PU is not detected and stop transmitting as soon as possible, preventing collisions and disruption of the SU service. The correct execution of the DSA procedure increases the amount of available bandwidth for the SUs, at the same time $5 \mathrm{G}$ networks require more bandwidth [4], [5].

While the current 3GPP standards do not support DSA, it already support complementary solutions like: license-assisted access (LTE-LAA/LTE-U) of unlicensed bands (mainly focused on the ISM $5 \mathrm{GHz}$ band also used by Wi-Fi, using Listen-Before-Talk (LBT), a form of CSMA); license-sharedaccess (LSA), where mobile carriers are either given timed authorizations to access the channel; standalone unlicensed 
access (MulteFire) [5], [6]. Spectrum regulatory agencies are also pushing the General Authorized Access (GAA) licenses, a mix of LSA and DSA, with tiers indicating access priorities and where lower tier users can access unused higher tier bands opportunistically [7].

The spectrum sharing procedure is usually divided into four steps: 1) sensing; 2) allocation; 3) access e 4) hand-off. In [1], the spectrum sensing techniques are grouped into three categories: a) non-collaborative, where the SUs detect the PU and avoid channels if a PU is detected, ideal for decentralized MAC; b) geolocation database, in which each SU sends the sensing results to an access point or radio-base station, that compares the result to the historical registries in the area, to find available bands; collaborative, which combines the results of multiple SUs sensing with a fusion algorithm that also considers the information of a database.

Widely used in the academy, the ns-3 [8] is an opensource discrete-event network simulator. When compared to other open-source simulators, the ns-3 offers additional Radio Access Technology (RAT) and multi-band models, including 802.11ax (Wi-Fi), 802.11 ad (WiGig), 802.16 (WiMAX), LTE (Long Term Evolution) and others. The ns- 3 community is very active and there is interest in increasing the ns-3 models functionality, allowing the experimentation and evaluation of new techniques used in 5G networks. The developers of the ns-3 LTE module are also working on a 5G NR model in [9], focusing on the new features (e.g. Bandwidth Part (BWP) and millimeter waves), but it still is under active development and under validation. However, until the writing of this paper, there was not yet any stable implementation of a cognitive cycle, that includes collaborative sensing algorithms and fusion for resource blocks allocation for LTE and 5G networks.

This work presents a set of adaptations and simulations results for the LTE module of the ns-3, implementing all the steps required for a generic cognitive cycle, that can be adapted for 5G networks. The implementation and validation of this module for the ns-3 simulator using the LTE stack serves as a base for further developments in a 3GPP [10] network. The collaborative sensing algorithms allows that the mobile network tower, known as evolved NodeB (eNB) in LTE and gigabit node $\mathrm{B}(\mathrm{gNB})$ in $5 \mathrm{G}$, to detect the PU presence on a channel based on reports from the mobile devices, known as User Equipment's (UEs).

The contributions expected from the current work to the 4G/5G research are the following: a) support the development of new resource scheduling algorithms and collaborative sensing fusion algorithms, by providing a simulator that implements a generic collaborative spectrum sensing cycle; b) support the development of new techniques to characterize the access behavior of the PU, allowing for the opportunistic use of licensed bands; c) evaluation of the impact of physical layer sensing mechanisms in the higher layers for different applications on both LTE and 5G networks.

This paper is organized as follows: Section II presents the specification and implementation of the cognitive cycle and show how its results are used by the resource scheduler.

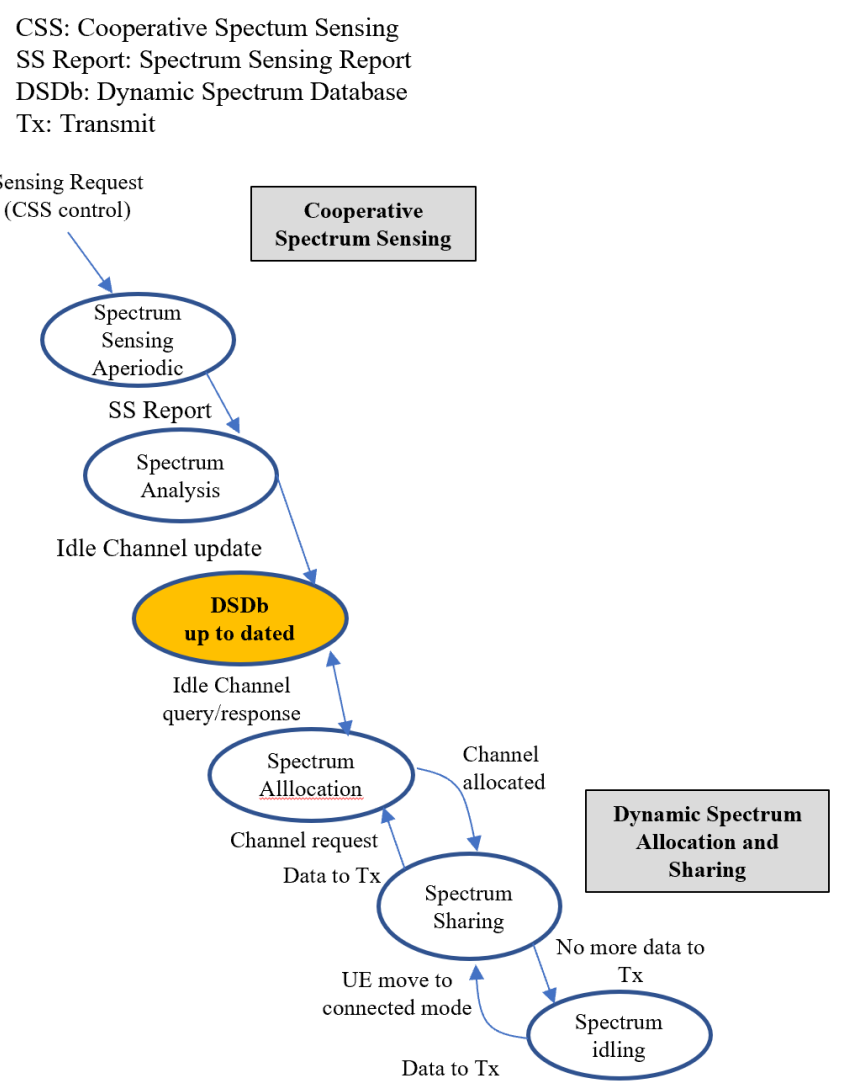

Fig. 1. Cognitive Cycle

Section III describes and analyses simulation results in a rural scenario. Finally, Section IV presents the conclusions and future work of this research.

\section{SPeCification AND IMPLEMENTATION OF THE Cognitive CyCLE}

The implementation of the Cognitive Cycle integrates Cooperative Spectrum Sensing (CSS) with centralized cooperation using a Fusion Center (FC), and using TVWS database approach (Geolocation Database-GDB). The cycles are presented in Fig. 1 are considered the basis for the scope of the simulator implementation, with the following considerations: (a) a set of UEs in the cell performs the present cycles independently; (b) UEs are always in connected mode; (c) UE provides Spectrum Sensing (SS) report in an aperiodic way triggered by the Cooperative Spectrum Sensing (CSS) report request issued by the CSS controller; (c) the request considers the Geolocation Database (GDB) information to define which UE and which TV channels needs to be sensed; (d) there is no use of CQI report for decisions taken by the Spectrum Analysis function (which would provide additional information in case distancebased detection probability curves are used).

The individual sensing of the UEs and reception of sensing results by the gNBs was implemented in the LtespectrumPhy class, that simulates the interaction between the physical layer and the channel of both UEs and 
gNBs. The reception method includes a call for the sensing function (Sense). The reception method for downlink control messages was modified to allow the reception of the spectrum sensing results and their forwarding to the upper layer.

The MAC layer of the UE was modified to include a function that assemblies and enqueues the sensing results control message. The MAC layer of the gNB was modified to include a function to receive control messages with sensing results and fuse the results of the sensing. Additionally, the eNB MAC function responsible for the subframe assembly and transmission was modified to call collaborative sensing fusion before the resource scheduling, and then to forward the fusion results to the scheduler.

Each of the gNB schedulers required modifications to receive a bitmap that carries the result of the collaborative sensing fusion, and is used to flag which Resource Block Groups (RBGs) are occupied by the sensed PU.

The cognitive cycle algorithm is described in Fig. 2. The control channel is assumed to be in a licensed channel without third-party interference. The data channel is assumed to be licensed by the PU. The steps start with the gNB transmitting a DCI and UCI with allocated resource blocks for the UEs, then the UEs transmit their control signaling, and repeating this process indefinitely. On step 4, the PU starts transmitting on the channel, the UE senses its presence and sends the control message flagging resource blocks or channels where it detected the PU. On step 6 the gNB fuses the sensing results of all UEs and then use the results in the next round, transmitting the DCI without scheduling the blocks or channel where the PU was detected. When the UEs stop detecting the PU, their sensing results will have no flagged block, and the gNB will start scheduling those blocks again.

As the ns-3 does not emulate the physical layer, the sensing algorithm implemented relies on simulated measurements of the Signal-to-Noise Ratio (SNR) values and/or the distance between the sender and receiver, along with a PU detection probability curve. The probability curve is produced by the real spectrum sensing algorithms on a link-level simulator and then loaded into ns-3 for system-level simulations in form of a JSON file loaded at runtime. One example of the PU detection probability table is shown in Fig. 3.

The sensing function calls a specialized function to execute the sensing procedure, that can be different depending on the use cases. A prototype sensing procedure function (sensingProcedure) that works for either SNR values or distance between the UE and PU was implemented as a reference. The sensingProcedure function verifies and interpolates the probability of detection for each $\mathrm{RB}$ or for groups of RBs. The interpolation uses either the individual or average SNR values of the RBGs, or the distance between the PU and UE. The Fig. 3 show an example of PU detection probability curve that serves as an input to the ns-3 simulator with a probability of false alarm $\mathrm{Pfa}=0.10$. The physical layer of the gNBs forwards the sensing messages received to the gNB MAC.

At the UEs MAC layer, two versions of sensing results notification are available, one using the data channel and other using the control channel. The sensing report contains the number of the frame and subframe in which the sensing was performed, a flag indicating if the PU was present, along with two bitmaps, one indicating the sensing on each RBG and other indicating false positives (when a PU was not present but was detected) and false negatives (when a PU was present but was not detected). Only the sensing bitmap is used for the sensing itself, while the rest of the data is used for statistics. The eNB MAC receives the sensing reports from either the control or data channel saving the report in a registry for later use. During the subframe indication and before triggering the MAC scheduler, the collaborative sensing fusion is called (mergeSensingReports) using the reports registry. During the fusion, the TVWS database, loaded along with the simulation parameters from a JSON file, can also be used to mask which channels are free or occupied based on the central frequency and bandwidth of the channel, along with the transmission period and the duty cycle of the PU occupying that channel. The result of the fusion is a bitmap, used by the schedulers to ignore RBGs where the PU presence was detected.

\section{Simulation Results}

To validate the cognitive cycle implementation, the simulation was done in a scenario, as shown in Fig. 4 with the following parameters:

a) $20 \mathrm{MHz}$ Additive White Gaussian Noise (AWGN) channel, broken into $4 \times 5 \mathrm{MHz}$ channels, noise floor of -174 $\mathrm{dBm} / \mathrm{Hz}, \mathrm{FSPL}$ pathloss model. In real scenarios, the TVWS has $24 \mathrm{MHz}$ bandwidth divided into 3 x $8 \mathrm{MHz}$ (Europe) or $4 \mathrm{x}$ $6 \mathrm{MHz}$ (Brazil) data channels. In the simulation, to abstract the physical layer and keep compatibility with the LENA module, the $20 \mathrm{MHz}$ bandwidth emulates the $24 \mathrm{MHZ}$ TVWS channel and translates 100 LTE RBs, that were grouped into 50 RBGs of size 2 (also required changes to the DCI Type 0 , used in the NS-3/LTE model). Those RBGs were then grouped into 4 subchannels of 3 x 13 RBGs $(5.2 \mathrm{MHz})$ and 1 x 11 RBGs (4.4 MHz);

b) the channel is located in band $5(869 \mathrm{MHz})$. gNB transmits with $53 \mathrm{dBm}$ and is equipped with $9 \mathrm{dBi}$ gain antennas, resulting in a $50 \mathrm{~km}$ radius cell. UEs transmit with $23 \mathrm{dBm}$ and is equipped with $9 \mathrm{dBi}$ gain antennas. The PUs transmit with $40 \mathrm{dBm}$. The UEs are placed at a fixed $30 \mathrm{~km}$ radius from the PU0, while PUs1-3 are placed at a fixed 40 $\mathrm{km}$ from the PU0. The PU0 is $35 \mathrm{~km}$ away from the gNB. This scenario characterizes a rural area with a $50 \mathrm{~km}$ cell, one gNB and 4 PUs channels;

c) all the spectrum sensing algorithm used in the simulation is WIBA [12], collecting data from 10 UEs. The PU detection probability curves used are shown in Fig. 3. To evaluate the different possibilities, at the fusion center in the gNB, 4 different fusion algorithms were implemented (OR, 2-of-10, 3-of-10, 4-of-10).

The results for different fusion algorithms of the simulated scenario are shown in Table I. False positives are the ratio 


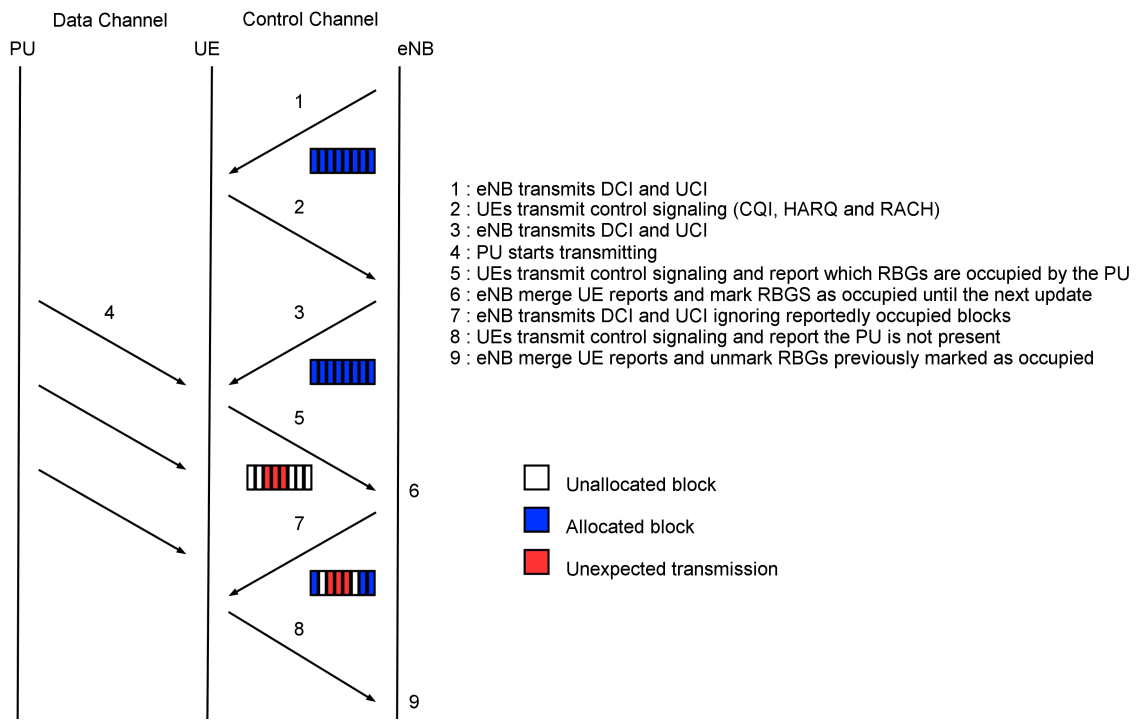

Fig. 2. Cognitive Cycle Algorithm

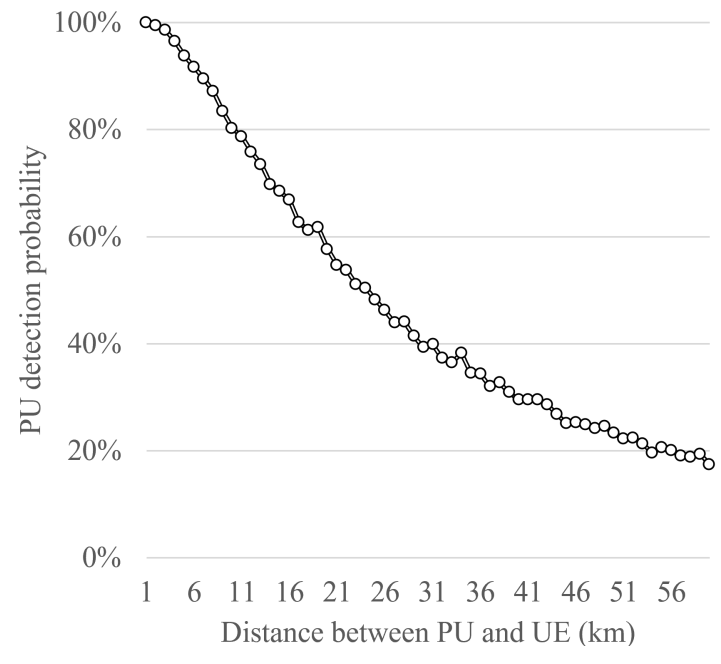

Fig. 3. PU detection probability vs. distance [11]

of subframes where no PU present but was detected and the number of subframes the PU was not present. False negatives show the ratio of subframes where a PU was present but was not detected and the number of subframes that the PU was present. False positives result in wasted radio resources while false negatives result in interference to the PU. The false positives for all channels are below $10 \%$ for all the types of fusion rules while false negatives increase with higher detection thresholds.

The simulation results of the cognitive cycle are shown in Fig. 5, based on the topology shown in Fig. 4, that contains 10 UEs, 1 eNB and 4 PUs, one for each channel. The first lines of Fig. 5 show the perceived PSD of each of the PUs in their respective channel. The second line show the individual SNR levels of each of the UEs for each of the four channels. The third line show the results of the RBGs scheduling, where

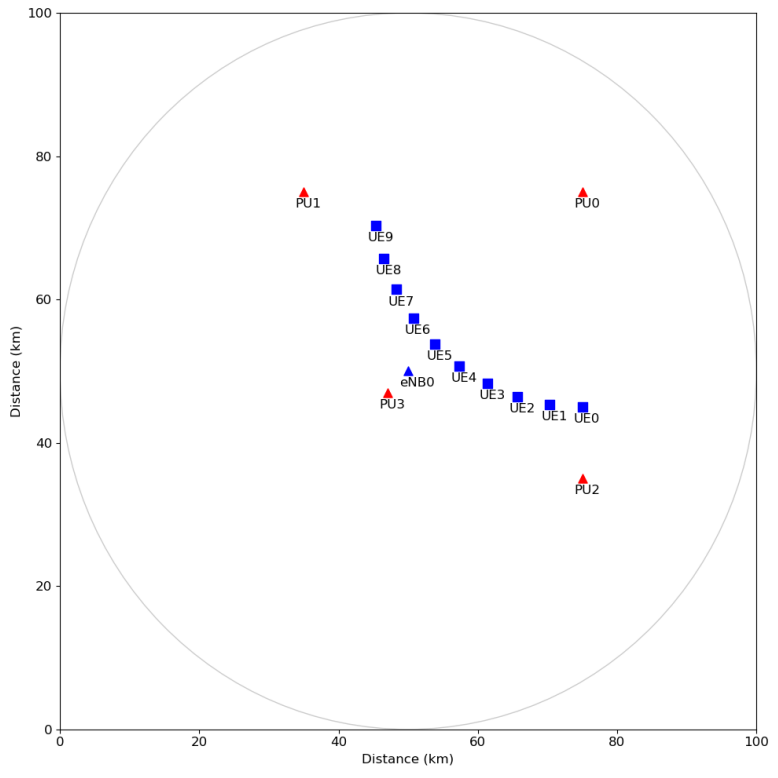

Fig. 4. Topology of the simulation scenario for rural areas

blank spaces are unused spectrum, blue spaces are scheduled spectrum and red spaces are avoided spectrum. The third line directly reflects the collaborative sensing fusion behavior, being capable of increasing or decrease the false alarms and false negatives. As false negatives implicate in collisions with the PU, the main target with the collaborative sensing fusion should be decreasing false negatives while keeping false positives low.

\section{CONClusions AND Future Work}

This paper presented a tool that can be used to develop and test different cognitive radio techniques to promote opportunistic use of the spectrum integrated into the LTE network stack 

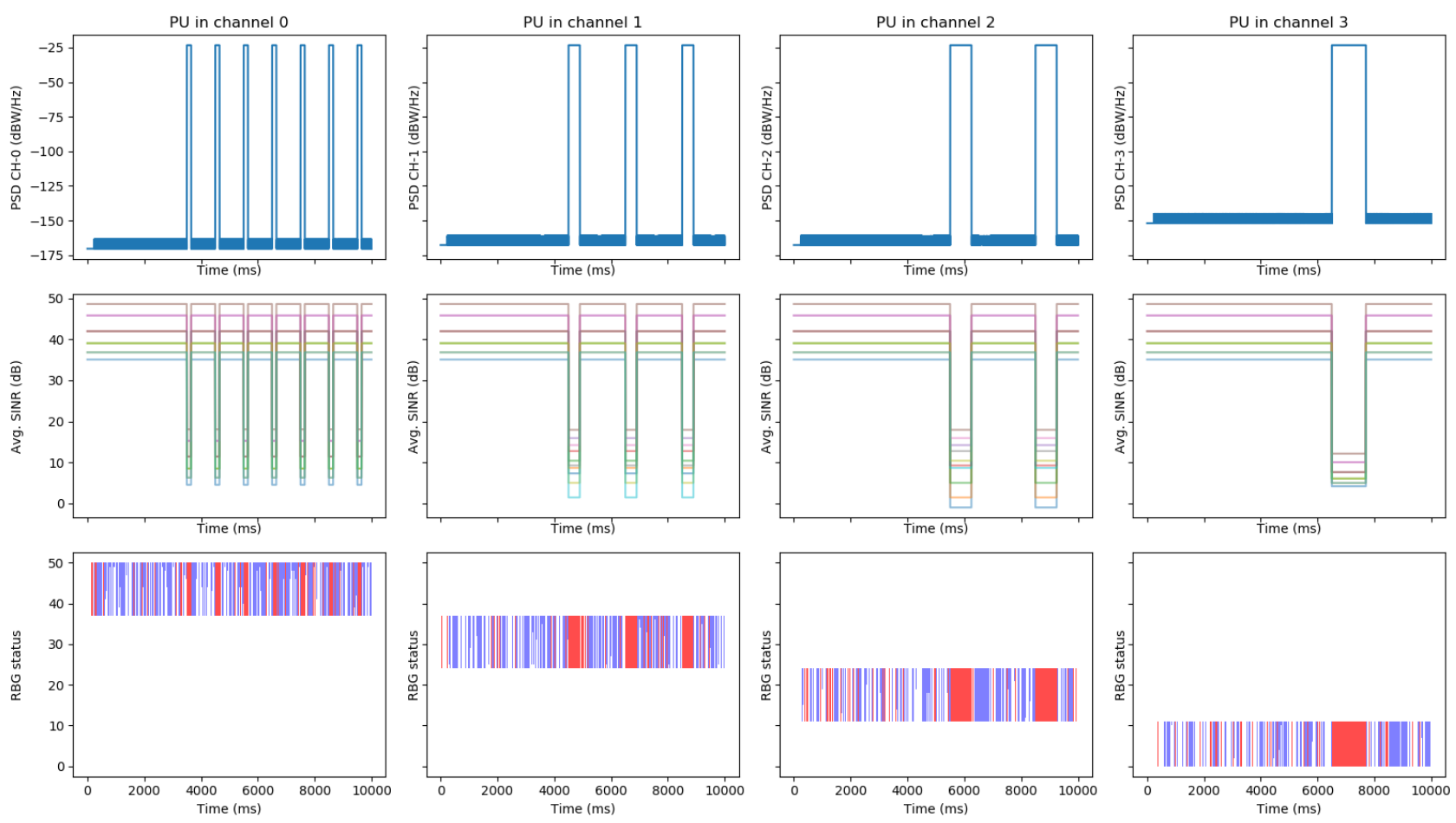

Fig. 5. Results of the collaborative sensing using OR fusion and radio resource scheduling for the simulated scenario.

\begin{tabular}{|c|c|c|c|c|c|c|c|c|}
\hline & \multicolumn{3}{|c|}{ Channel 0 } & \multicolumn{2}{c|}{ Channel 1 } & \multicolumn{2}{c|}{ Channel 2 } & \multicolumn{2}{|c|}{ Channel 3 } \\
Fusion & $\begin{array}{c}\text { False } \\
\text { Positives } \\
(\%)\end{array}$ & $\begin{array}{c}\text { False } \\
\text { Negatives } \\
(\%)\end{array}$ & $\begin{array}{c}\text { FP } \\
(\%)\end{array}$ & $\begin{array}{c}\text { FN } \\
(\%)\end{array}$ & $\begin{array}{c}\text { FP } \\
(\%)\end{array}$ & $\begin{array}{c}\text { FN } \\
(\%)\end{array}$ & $\begin{array}{c}\text { FP } \\
(\%)\end{array}$ & $\begin{array}{c}\text { FN } \\
(\%)\end{array}$ \\
\hline OR & 9.2 & 0.4 & 9.9 & 0.3 & 9.3 & 0.0 & 9.7 & 0.0 \\
\hline 2-of-10 & 0.4 & 5.0 & 0.4 & 2.2 & 0.4 & 0.3 & 0.5 & 0.1 \\
\hline 3-of-10 & 0.1 & 17.9 & 0.0 & 3.9 & 0.0 & 3.9 & 0.0 & 0.3 \\
\hline 4-of-10 & 0.0 & 38.1 & 0.0 & 27.3 & 0.0 & 14.4 & 0.0 & 2.8 \\
\hline
\end{tabular}

TABLE I

COLLABORATIVE SENSING RESULTS FOR THE SIMULATED SCENARIO.

or a $5 \mathrm{G}$ physical layer implemented in the ns-3 simulator. The cognitive cycle was validated in a scenario for rural areas with a $50 \mathrm{~km}$ cell, 10 UEs, 4 PUs and using the WIBA sensing algorithm. As future work it is planned to optimize the MAC Cognitive layer regarding the fusion algorithm at the gNB, that can exploit a multi-frame approach and the also the use of deep learning algorithms for RGBs scheduling. Also, the verification of other spectrum sensing algorithms may allow the exploration of state-of-the-art techniques in the Cognitive MAC Layer and optimize its different functionalities.

\section{ACKNOWLEDGMENTS}

This work was supported by the 5G-Range project (www.5g-range.eu), approved in the 4th EU-BR call in TICs, financed by the CTIC/RNP/MCTIC and by the European Commission Research and Innovation Action H2020-EUB2017 EU-BRAZIL Joint Call Grant ICT-777137.

\section{REFERENCES}

[1] F. Hu, B. Chen, and K. Zhu, "Full spectrum sharing in cognitive radio networks toward 5g: A survey," IEEE Access, vol. 6, pp. 15 754-15 776, 2018.

[2] V. Valenta, Z. Fedra, R. Marsalek, G. Baudoin, and M. Villegas, "Towards cognitive radio networks: Spectrum utilization measurements in suburb environment," in 2009 IEEE Radio and Wireless Symposium. IEEE, jan 2009.

[3] R. Beckman, K. Channakeshava, F. Huang, V. S. A. Vullikanti, A. Marathe, M. V. Marathe, and G. Pei, "Implications of dynamic spectrum access on the efficiency of primary wireless market," in 2010 IEEE Symposium on New Frontiers in Dynamic Spectrum (DySPAN). IEEE, apr 2010.

[4] A. O. Arafat, A. Al-Hourani, N. S. Nafi, and M. A. Gregory, "A survey on dynamic spectrum access for LTE-advanced," Wireless Personal Communications, vol. 97, no. 3, pp. 3921-3941, aug 2017.

[5] M. Szydelko and M. Dryjanski, "3gpp spectrum access evolution towards 5g," EAI Endorsed Transactions on Cognitive Communications, vol. 3, no. 10, p. 152184, feb 2017.

[6] S. Yrjl and H. Kokkinen, "Licensed shared access evolution enables early access to $5 \mathrm{~g}$ spectrum and novel use cases," EAI Endorsed Transactions on Wireless Spectrum, vol. 3, no. 12, p. 153463, dec 2017.

[7] S. Basnet, B. A. Jayawickrama, Y. He, E. Dutkiewicz, and M. D. Mueck, "Opportunistic access to PAL channel for multi-RAT GAA transmission in spectrum access system," in 2017 IEEE 85th Vehicular Technology Conference (VTC Spring). IEEE, jun 2017.

[8] NSNAM, "ns-3 network simulator," march 2019, https://www.nsnam.org/.

[9] CTTC, "The first release of 5g-lena is available," feb 2019, http://www.cttc.es/the-first-release-of-5g-lena-is-available/.

[10] A. Sultan, “21.915 release 15," Tech. Rep., January 2019, https://portal.3gpp.org/desktopmodules/Specifications/SpecificationDetails .aspx?specificationId $=3389$.

[11] 5G-Range, "Spectrum sensing to complement databases," Tech. Rep., oct 2018, http://5g-range.eu/wp-content/uploads/2018/04/D4.2Spectrum-Sensing-to-Complement-Databases.pdf.

[12] H. Saarnisaari and J. Vartiainen, "Spectrum window based signal detection at low SNR," in 2018 International Conference on Military Communications and Information Systems (ICMCIS). IEEE, may 2018. 